\title{
Continuous Abstraction of Nonlinear Systems using Sum-of-Squares Programming
}

\author{
Stanley W. Smith, He Yin and Murat Arcak
}

\begin{abstract}
We present a control design procedure for nonlinear control systems in which we represent a potentially high dimensional system with a low dimensional continuous-state abstraction. The abstraction generates a reference which the original system follows with a low level controller. We propose sum-of-squares programming as a tool to design this controller and to provide an upper bound on the relative error between the system and its abstraction. We compute the low level controller simultaneously with a simulation function that gives the boundedness guarantee for the relative error.
\end{abstract}

\section{INTRODUCTION}

An important task for cyber-physical systems is to design controllers that perform complex tasks while providing safety guarantees. Formal methods approaches [1] allow one to specify desired behaviors and then compute control strategies that achieve them, but are often limited to systems with small state dimension. Thus, it is beneficial to represent the system of interest with a lower dimensional model that is amenable to such approaches. A rigorous framework for such reduction is the notion of a continuous-state abstraction [2], henceforth called a continuous abstraction, which provides a low level controller to ensure boundednesss of the error between the system and its abstraction as well as error bounds. In [2], the authors also provide conditions under which the error converges to zero, certified by a Lyapunov-like function referred to as a simulation function. This is extended in [3], [4] to interconnected control systems, where an additional condition on the interconnection topology is required, and the simulation function is constructed in a compositional manner using small-gain type reasoning and dissipativity properties of the subsystems, respectively.

In [5], an explicit connection is made between the aforementioned conditions and the existence of an invariant manifold on which the full system model reduces to the abstraction. In addition, [5] and [6] show that one can relax this invariance condition, resulting in a disturbance term in the dynamics relative to the manifold. This relaxation broadens the applicability of continuous abstraction; however, the boundedness analysis in [5] and [6] for the error system relies on an incremental dissipativity condition that may be hard to establish in practice.

Funded in part by an NDSEG Graduate Fellowship, the Air Force Office of Scientific Research grant FA9550-18-1-0253, and the Office of Naval Research grant N00014-18-1-2209.

The first two authors contributed equally to this work.

S. W. Smith and M. Arcak are with the Department of Electrical Engineering and Computer Sciences, University of California, Berkeley \{swsmth, arcak\}@eecs.berkeley.edu.

$\mathrm{H}$. Yin is with the Department of Mechanical Engineering, University of California, Berkeley he_yin@berkeley.edu .
In this paper, we do not require invariance or incremental dissipativity conditions; instead, we formulate the search for a low level controller and a simulation function for error bounds as a sum-of-squares (SOS) optimization problem. We approximate the system dynamics with a polynomial model and assume that the abstract state and input are restricted to semi-algebraic constraint sets. These sets and the model are then used as an input to our SOS optimization problem which, when feasible, returns a corresponding error bound. If the resulting error bound is too large, the constraint sets can be restricted further in an iterative procedure outlined in Section II-C.

Related publications other than those from the continuous abstraction literature include a modular control design approach to motion planning in [7], which consists of a planning layer and a tracking layer, and obtains an upper bound on the tracking error using Hamilton-Jacobi reachability analysis. An extension in [8] shows how this may also be done with SOS optimization. In [9], SOS optimization is used to construct a function that bounds the error between a high fidelity model and a low fidelity model. We note that these works focus on motion planning applications where the zero-error manifold is a subspace with a particular structure, whereas we allow any manifold to be used depending on the application. For example, in this paper we include a platooning application where some of the desired states are a translation of the abstract states, and in prior work [6] we included a temperature control application where multiple states are aggregated into one abstract state. In [10], a bound is computed for the relative distance between the closed-loop system trajectory and a nominal trajectory that is given as a time series. However, a large number of bounds need to be precomputed for different nominal trajectories to consider all the possible maneuvers of the system.

The paper is organized as follows. In Section II the framework for continuous abstraction is reviewed, and methods for computing the error bounds and low level controllers are proposed. In Section III and IV] our method is applied to two nonlinear system examples. Section $\mathrm{V}$ summarizes the results.

Notation: $\mathcal{C}^{1}$ is the set of differentiable functions whose derivative is continuous. For $\xi \in \mathbb{R}^{n}, \mathbb{R}[\xi]$ represents the set of polynomials in $\xi$ with real coefficients, and $\mathbb{R}^{m}[\xi]$ and $\mathbb{R}^{m \times p}[\xi]$ denote all vector and matrix valued polynomial functions. The subset $\Sigma[\xi]:=\left\{p=p_{1}^{2}+p_{2}^{2}+\ldots+p_{M}^{2}\right.$ : $\left.p_{1}, \ldots, p_{M} \in \mathbb{R}[\xi]\right\}$ of $\mathbb{R}[\xi]$ is the set of SOS polynomials in $\xi$. For $\gamma \in \mathbb{R}$, and continuous $V: \mathbb{R}^{n} \rightarrow \mathbb{R}$, we denote the 
sublevel set as

$$
\Omega_{\gamma}^{V}:=\left\{e \in \mathbb{R}^{n}: V(e) \leq \gamma\right\} .
$$

\section{Continuous Abstraction}

We consider control affine systems of the form

$$
\Sigma: \quad \dot{x}(t)=f(x(t))+g(x(t)) u(t),
$$

where $x(t) \in \mathbb{R}^{n}, u(t) \in \mathbb{R}^{m}, f: \mathbb{R}^{n} \rightarrow \mathbb{R}^{n}$, and $g:$ $\mathbb{R}^{n} \rightarrow \mathbb{R}^{n} \times \mathbb{R}^{m}$. We refer to the original system (2) as the concrete system. To facilitate the control design procedure, we represent (2) with a continuous abstraction

$$
\hat{\Sigma}: \quad \dot{\hat{x}}(t)=\hat{f}(\hat{x}(t))+\hat{g}(\hat{x}(t)) \hat{u}(t),
$$

where $\hat{x}(t) \in \mathbb{R}^{\hat{n}}, \hat{u}(t) \in \mathbb{R}^{\hat{m}}, \hat{f}: \mathbb{R}^{\hat{n}} \rightarrow \mathbb{R}^{\hat{n}}$, and $\hat{g}:$ $\mathbb{R}^{\hat{m}} \rightarrow \mathbb{R}^{\hat{n}} \times \mathbb{R}^{\hat{m}}$. Since the abstraction (3) typically has a smaller state dimension than the full system (2), we define a map $\pi: \mathbb{R}^{\hat{n}} \rightarrow \mathbb{R}^{n}$ which allows comparison of $x(t)$ and $\hat{x}(t)$. In particular, we want $\Sigma$ and $\hat{\Sigma}$ to remain within close proximity to the manifold

$$
x(t)=\pi(\hat{x}(t))
$$

for all $t \geq 0$. Thus, we may interpret $\pi(\hat{x}(t))$ as generating a reference signal for $\Sigma$ to track. The examples in Sections III and IV illustrated physically meaningful choices of $\pi$.

The abstract control $\hat{u}(t)$ could be designed formally so that (3) satisfies a high level objective expressible in, for example, Signal Temporal Logic (STL) [11]. Such objectives can be achieved with a numerical optimization-based approach, such as model predictive control (MPC) [12]. However, since such methods do not scale to large systems, a shrunken state dimension for (3) is beneficial.

\section{A. The Error System}

To ensure boundedness of the error $e(t):=x(t)-\pi(\hat{x}(t))$, we incorporate a low level controller

$$
u(t)=\kappa(e(t), \hat{x}(t), \hat{u}(t))
$$

to be designed and note that $e(t)$ evolves according to

$$
\begin{aligned}
\dot{e}(t) & =f_{e}(e(t), \hat{x}(t), \hat{u}(t)) \\
& +g_{e}(e(t), \hat{x}(t)) \kappa(e(t), \hat{x}(t), \hat{u}(t)),
\end{aligned}
$$

where $f_{e}(e(t), \hat{x}(t), \hat{u}(t)):=$

$$
\begin{aligned}
& f(\pi(\hat{x}(t))+e(t))-\frac{\partial \pi}{\partial \hat{x}} \cdot(\hat{f}(\hat{x}(t))+\hat{g}(\hat{x}(t)) \hat{u}(t)), \\
& g_{e}(e(t), \hat{x}(t)):=g(\pi(\hat{x}(t))+e(t)) .
\end{aligned}
$$

To design the low level controller (5) and to provide error bounds, we make use of Proposition 1 below, which assumes that the abstract state and input are restricted to the sets $\hat{\mathcal{X}}$ and $\hat{\mathcal{U}}$, respectively. This assumption is satisfied by designing a control $\hat{u}$ which imposes constraints on $\hat{x}$ and $\hat{u}$.

Proposition 1: Given the error dynamics (6) with mappings $f_{e}: \mathbb{R}^{n} \times \mathbb{R}^{\hat{n}} \times \mathbb{R}^{\hat{m}} \rightarrow \mathbb{R}^{n}, g_{e}: \mathbb{R}^{n} \times \mathbb{R}^{\hat{n}} \rightarrow \mathbb{R}^{n}$, and $\gamma>0, \hat{\mathcal{X}} \subseteq \mathbb{R}^{\hat{n}}, \hat{\mathcal{U}} \subseteq \mathbb{R}^{\hat{m}}$, if there exists a $\mathcal{C}^{1}$ function $V: \mathbb{R}^{n} \rightarrow \mathbb{R}^{+}$, and $\kappa: \mathbb{R}^{n} \times \mathbb{R}^{\hat{n}} \times \mathbb{R}^{\hat{m}} \rightarrow \mathbb{R}^{m}$, such that

$$
\begin{aligned}
& V(0)=0 \text { and } V(e)>0 \text { for all } e \neq 0, \\
& \frac{\partial V}{\partial e} \cdot\left(f_{e}(e, \hat{x}, \hat{u})+g_{e}(e, \hat{x}) \kappa(e, \hat{x}, \hat{u})\right)<0, \\
& \quad \forall e, \hat{x}, \hat{u}, \text { s.t. } V(e) \geq \gamma, \hat{x} \in \hat{\mathcal{X}}, \hat{u} \in \hat{\mathcal{U}},
\end{aligned}
$$

then the sublevel set $\Omega_{\gamma}^{V}$, defined in 1 , is forward invariant. If, further, $V$ is radially unbounded, then $\forall e(0) \in \mathbb{R}^{n}, e(t)$ remains bounded and converges to $\Omega_{\gamma}^{V}$.

The set $\Omega_{\gamma}^{V}$ is an error bound for the error dynamics 6 , associated with the control law $\kappa$. If the low level control is subject to the constraints $u(t) \in \mathcal{U}$, where $\mathcal{U}:=\left\{u \in \mathbb{R}^{m}\right.$ : $\underline{u} \leq u \leq \bar{u}\}$, the symbol " $\leq$ " represents componentwise inequality, we augment the constraints (9), (10) and $V$ being radially unbounded with the set containment constraints: for all $i=1, \ldots, m$,

$$
\begin{aligned}
& \Omega_{\gamma}^{V} \subseteq\left\{e \in \mathbb{R}^{n}: \kappa_{i}(e, \hat{x}, \hat{u}) \leq \bar{u}_{i}\right\}, \quad \forall \hat{x} \in \hat{\mathcal{X}}, \hat{u} \in \hat{\mathcal{U}} \\
& \Omega_{\gamma}^{V} \subseteq\left\{e \in \mathbb{R}^{n}: \underline{u}_{i} \leq \kappa_{i}(e, \hat{x}, \hat{u})\right\}, \quad \forall \hat{x} \in \hat{\mathcal{X}}, \hat{u} \in \hat{\mathcal{U}}
\end{aligned}
$$

To achieve a less conservative bound, we formulate a highlevel optimization problem with the volume of $\Omega_{\gamma}^{V}$ as the objective function and (9) to (12) as constraints. We denote the problem as $\boldsymbol{o p t}\left(f_{e}, \boldsymbol{g}_{e}, \gamma, \overrightarrow{\mathcal{X}}, \hat{\mathcal{U}}, \mathcal{U}\right)$ :

$$
\begin{aligned}
& \min _{V, \kappa} \operatorname{volume}\left(\Omega_{\gamma}^{V}\right), \\
& \text { s.t. }(9)-(12) \text {, and } V \text { is radially unbounded. }
\end{aligned}
$$

\section{B. Sum-of-Squares formulation of the optimization problem}

To formulate opt above as a SOS problem, assume that the sets $\hat{\mathcal{X}}$ and $\hat{\mathcal{U}}$ are semi-algebraic sets:

$$
\begin{aligned}
& \hat{\mathcal{X}}:=\left\{\hat{x} \in \mathbb{R}^{\hat{n}}: \hat{p}_{1}(\hat{x}) \leq 0\right\}, \\
& \hat{\mathcal{U}}:=\left\{\hat{u} \in \mathbb{R}^{\hat{m}}: \hat{p}_{2}(\hat{u}) \leq 0\right\},
\end{aligned}
$$

where the polynomial functions $\hat{p}_{1}$ and $\hat{p}_{2}$ are chosen based on the state and input constraints we enforced in the formal synthesis for the abstract system. We also assume that $f_{e}(e, \hat{x}, \hat{u}) \in \mathbb{R}^{n}[(e, \hat{x}, \hat{u})]$ and $g_{e} \in \mathbb{R}^{n \times m}[(e, \hat{x})]$.

Using sum-of-squares relaxation for non-negativity, and the generalized S-procedure [13] for set containment, we give the following SOS optimization problem denoted as $\operatorname{sosopt}\left(f_{e}, g_{e}, \gamma, \hat{p}_{1}, \hat{p}_{2}, \underline{u}, \bar{u}\right)$ :

$$
\begin{aligned}
\min _{V, \kappa, s_{i}, s_{k}^{j}} & \operatorname{volume}\left(\Omega_{\gamma}^{V}\right) \\
\text { s.t. } & s_{i} \in \Sigma[(e, \hat{x}, \hat{u})], \forall i=1, \ldots, 3, \\
& s_{k}^{j} \in \Sigma[(e, \hat{x}, \hat{u})], \forall j=1, \ldots, m, \forall k=4, \ldots, 9, \\
& \kappa \in \mathbb{R}^{m}[(e, \hat{x}, \hat{u})], V(e)-L_{1} \in \Sigma[e], \\
& -\frac{\partial V}{\partial e} \cdot\left(f_{e}+g_{e} \cdot \kappa\right)-L_{2}+s_{1} \cdot \hat{p}_{1}+s_{2} \cdot \hat{p}_{2} \\
& -s_{3} \cdot(V-\gamma) \in \Sigma[(e, \hat{x}, \hat{u})] \\
& \bar{u}_{i}-\kappa_{i}+s_{4}^{j} \cdot(V-\gamma)+s_{5}^{j} \cdot \hat{p}_{1} \\
& +s_{6}^{j} \cdot \hat{p}_{2} \in \Sigma[(e, \hat{x}, \hat{u})], \forall j=1, \ldots, m, \\
& \kappa_{i}-\underline{u}_{i}+s_{7}^{j} \cdot(V-\gamma)+s_{8}^{j} \cdot \hat{p}_{1}
\end{aligned}
$$




$$
+s_{9}^{j} \cdot \hat{p}_{2} \in \Sigma[(e, \hat{x}, \hat{u})], \forall j=1, \ldots, m .
$$

In the above formulation, SOS polynomials $s_{i}$ and $s_{k}^{j}$ are multipliers used in the generalized S-procedure, and $L_{1}=\epsilon_{1} e^{\prime} e, L_{2}=\epsilon_{2}[e ; \hat{x} ; \hat{u}]^{\prime}[e ; \hat{x} ; \hat{u}]$ with $\epsilon_{1}$ and $\epsilon_{2}$ small positive number on the order of $10^{-6}$. Note that sosopt is a nonconvex problem, since the decision variables $\frac{\partial V}{\partial x}$ and $\kappa, s$ and $V$ multiply with each other. To tackle this problem, we decompose it into two tractable subproblems to iteratively search between the simulation function $V$ and multipliers and the control law $s_{i}, s_{k}^{j}, \kappa$.

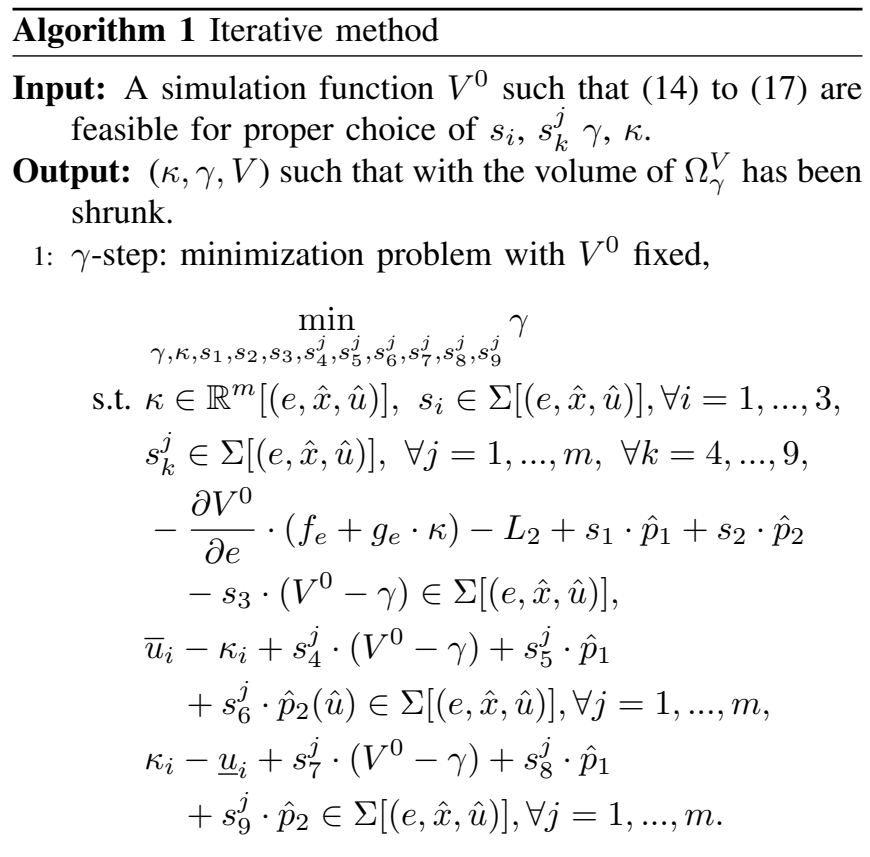

2: $V$-step: feasibility problem over decision variables $V, s_{1}, s_{2}, s_{5}^{j}, s_{6}^{j}, s_{8}^{j}, s_{9}^{j}, s_{10}$ :

$$
\begin{aligned}
& \left(s_{10}-\epsilon_{3}\right), V-L_{1} \in \Sigma[e], s_{1}, s_{2} \in \Sigma[(e, \hat{x}, \hat{u})], \\
& s_{k}^{j} \in \Sigma[(e, \hat{x}, \hat{u})], \forall j=1, \ldots, m, \forall k=5,6,8,9, \\
& \quad-s_{10} \cdot\left(V^{0}-\gamma^{*}\right)+\left(V-\gamma^{*}\right) \in \Sigma[e], \\
& -\frac{\partial V}{\partial e} \cdot\left(f_{e}+g_{e} \cdot \kappa^{0}\right)-L_{2}+s_{1} \cdot \hat{p}_{1}+s_{2} \cdot \hat{p}_{2} \\
& \quad-s_{3}^{0} \cdot\left(V-\gamma^{*}\right) \in \Sigma[(e, \hat{x}, \hat{u})], \\
& \bar{u}_{i}-\kappa_{i}^{0}+s_{4}^{j, 0} \cdot\left(V-\gamma^{*}\right)+s_{5}^{j} \cdot \hat{p}_{1} \\
& \quad+s_{6}^{j} \cdot \hat{p}_{2} \in \Sigma[(e, \hat{x}, \hat{u})], \forall j=1, \ldots, m, \\
& \kappa_{i}^{0}-\underline{u}_{i}+s_{7}^{j, 0} \cdot\left(V-\gamma^{*}\right)+s_{8}^{j} \cdot \hat{p}_{1} \\
& \quad+s_{9}^{j} \cdot \hat{p}_{2} \in \Sigma[(e, \hat{x}, \hat{u})], \forall j=1, \ldots, m .
\end{aligned}
$$

The $\gamma$-step fixes the simulation function $V$ to be $V^{0}$ computed from the $V$-step of the previous iteration, and tries to find the smallest $\Omega_{\gamma}^{V^{0}}$ by minimizing $\gamma$ over the control law and multipliers. Since $s_{3}$ and $\gamma$ multiply with each other, the minimization problem is a bilinear problem and can be effectively solved by bisecting $\gamma$.

In the optimization problem of the $V$-step, $\gamma^{*}, \kappa^{0}, s_{3}^{0}, s_{4}^{j, 0}$ and $s_{7}^{j, 0}$ are obtained from the $\gamma$-step, and $\epsilon_{3}$ the small positive number on the order of $10^{-6}$ ensures that $s_{10}$ can't take the value of zero. The constraint (18) enforces the error bound $\Omega_{\gamma^{*}}^{V}$ certified by the $V$-step of current iteration to be contained in $\Omega_{\gamma^{*}}^{V^{0}}$. After the $\gamma$-step, the constraints of the $\gamma$-step are active for $V^{0}$. In the $V$-step, a new feasible $V$ is computed, which is the analytic center of linear matrix inequality constraints. Thus the feasibility problem in the $V$-step pushes $V$ away from the constraints, and gives the next $\gamma$-step more freedom to decrease $\gamma$.

The input to Algorithm 1 is a feasible initial guess for $V^{0}$. One candidate might be a Lyapunov function $\bar{V}$ obtained by solving Lyapunov equations using the linearized error dynamics with LQR controllers. However, $\bar{V}$ may be too coarse to be feasible for the constraints (14) to (17), which are required to hold on a large set of $\mathcal{X}$ and $\mathcal{U}$. Here, we propose an algorithm for initializing $V^{0}$, the idea of which is to enforce the constraints on small subsets of $\hat{\mathcal{X}}, \hat{\mathcal{U}}$, at the beginning and gradually expand the subsets while maintaining feasibility by bisearching between $V$ and $\kappa$.

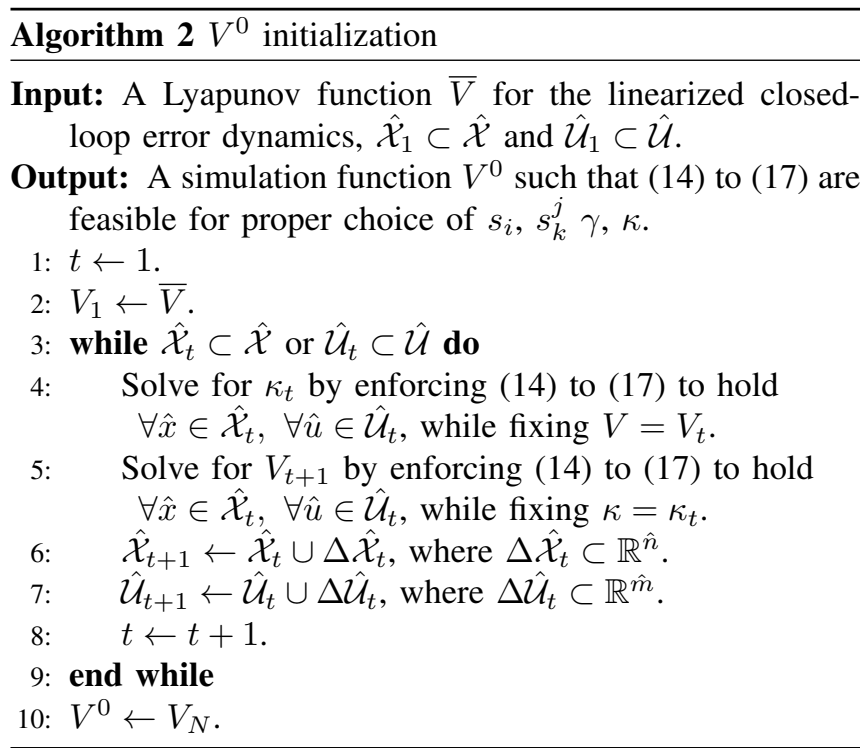

\section{Outline of the Design Procedure}

We now outline our overall control design process. In particular, if the error bound obtained from the procedure in Section III-B is too large, further modification to the controller for the abstraction will be necessary. We propose using the following heuristic approach to converge on an allowable error bound:

(i) Select an abstraction $\hat{\Sigma}$ and map $\pi(\cdot)$ based on the application. Then, design a control $\hat{u}$ for $\hat{\Sigma}$ which constrains $\hat{x}(t)$ and $\hat{u}(t)$ to the respective sets $\hat{\mathcal{X}}$ and $\hat{\mathcal{U}}$ (using, e.g., an MPC-based approach).

(ii) Complete the procedure outlined in Section II-B resulting in a low level controller $\kappa(e(t), \hat{x}(t), \hat{u}(t))$ for $\Sigma$ and a bound on the error $\Omega_{\gamma}^{V}$.

(iii) If step (ii) fails to be feasible, or if the error bound is too large, return to step $(i)$ and tighten the constraints on $\hat{x}(t)$ and $\hat{u}(t)$ by shrinking the sets $\hat{\mathcal{X}}$ and $\hat{\mathcal{U}}$. Repeat the process until the error bound is sufficiently small. 
To illustrate this procedure, we provide two examples in Sections III] and IV in which a suitable error bound was obtained by iterating through steps $(i)$ - (iii). In both examples, only a few iterations were necessary.

\section{Vehicle Platoon EXAMPLE}

\section{A. Model}

In this section we apply the previous approach to a vehicle platoon application adapted from [5]. We consider the longitudinal dynamics of two vehicle platoons, where the preceding platoon is comprised of a leader vehicle and $n$ follower vehicles, and the latter of one leader vehicle and $m$ follower vehicles (see Figure 1 where $n=m=1$ ). The task is to formally synthesize a centralized controller for an abstraction of the platoon, with the goal of achieving a high level objective expressed in STL. A low level controller as in Section $\mathrm{II}$ is then designed to minimize the error bound between the platoon and its abstraction.

The longitudinal dynamics of the leader vehicle are

$$
\dot{x}_{a}=v_{a}, \quad \dot{v}_{a}=-v^{0}+v_{a}-\rho \cdot\left(v_{a}\right)^{2} / M+u_{a},
$$

where $x_{a}$ and $v_{a}$ are position and velocity, respectively, $v^{0}$ is a nominal velocity, $\rho$ is an air drag coefficient, $M$ is the vehicle mass, and $u_{a}$ is the control input to be designed. To improve readability, the time arguments for the variables of differential equation models are omitted in the examples. Furthermore, we note that the quadratic air drag term was not included in [5], which specialized to affine systems. The follower vehicles are governed by a simple vehicle-following model

$$
\begin{aligned}
& \dot{x}_{1}=v_{1}, \\
& \dot{v}_{1}=v^{0}-v_{1}-\rho \cdot\left(v_{1}\right)^{2} / M+g\left(x_{a}-x_{1}\right), \\
& \dot{x}_{i}=v_{i} \\
& \dot{v}_{i}=v^{0}-v_{i}-\rho \cdot\left(v_{i}\right)^{2} / M+g\left(x_{i-1}-x_{i}\right),
\end{aligned}
$$

for $i=2, \ldots, n$, where $g: \mathbb{R} \rightarrow \mathbb{R}$ is a monotone increasing function. The function $g(\cdot)$ models a unilateral spring force acting on vehicle $i$. Its purpose is to preserve an inter-vehicle spacing of $\ell \in \mathbb{R}$ within the platoon, and thus $g(\ell)=0$.

The second vehicle platoon has the same dynamics as the first; however, as a safety measure we also model a spring force between the $n$th follower vehicle and platoon leader $b$ :

$$
\begin{aligned}
& \dot{x}_{b}=v_{b}, \\
& \dot{v}_{b}=-v_{b}+v^{0}-\rho \cdot\left(v_{b}\right)^{2} / M+g\left(x_{n}-x_{b}\right)+u_{b} .
\end{aligned}
$$

We note that as long as $g(\cdot)$ is polynomial, the platoon dynamics are polynomial, and are therefore amenable to SOS programming. For simplicity, we use a linear spring force

$$
g(s)=k \cdot(s-\ell)
$$

where $k \in \mathbb{R}$ is a spring constant. We note that the overall platoon model may be represented compactly as in (2).

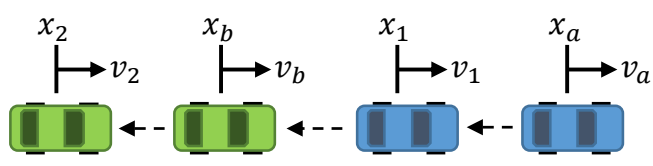

Fig. 1: Two platoons of vehicles with leader vehicles $a$ and $b$ and follower vehicles 1 and 2 . The position and velocity of each vehicle is labelled.

\section{B. Abstraction}

To obtain an abstract model of the platoon, we consider only the dynamics of the two leader vehicles

$$
\begin{aligned}
& \dot{\hat{x}}_{a}=v_{a}, \quad \dot{\hat{v}}_{a}=-\hat{v}^{0}+\hat{v}_{a}-\rho \cdot\left(\hat{v}_{a}\right)^{2} / M+\hat{u}_{a}, \\
& \dot{\hat{x}}_{b}=\hat{v}_{b}, \quad \dot{\hat{v}}_{b}=-\hat{v}^{0}+\hat{v}_{b}-\rho \cdot\left(\hat{v}_{b}\right)^{2} / M+\hat{u}_{b},
\end{aligned}
$$

for which we will formally synthesize a control strategy. We note, in particular, that this model ignores the spring force in (21), as the position of vehicle $n$ is not accessible. This effectively introduces a disturbance into the error dynamics (6), which is compensated for by the interface (5) - for further details see [5]. Lastly, we define the manifold (4) as follows

$$
\begin{aligned}
x_{a} & =\hat{x}_{a}, \quad x_{i}=\hat{x}_{a}-i \ell, i=1, \ldots, n, \\
v_{a} & =v_{1}=\cdots=v_{n}=\hat{v}_{a}, \\
x_{b} & =\hat{x}_{b}, \quad x_{n+i}=\hat{x}_{b}-i \ell, i=1, \ldots, m, \\
v_{b} & =v_{n+1}=\cdots=v_{n+m}=\hat{v}_{b},
\end{aligned}
$$

which is affine, and therefore amenable to our approach. Physically, this manifold means that 1 ) the leader vehicles match their respective reference positions, with all follower vehicles leaving $\ell$ meters of distance in front of them, and 2) all vehicles within each platoon match their respective reference velocity.

\section{Specification: Platoon Separation}

We consider a two platoons of vehicles each with one leader and one follower vehicle $(n=m=1)$. A common control objective for platooning is a gap widening maneuver, with the goal of allowing another vehicle to merge into the lane occupied by the platoon. This objective may be expressed in STL as follows

$$
\square\left(\varphi \wedge\left(S \Longrightarrow \diamond_{[0, T]} \psi\right)\right)
$$

Here, the $\square$ and $\diamond$ symbols are the "always" and "eventually" operators; thus, $\varphi$ expresses state constraints which must be satisfied at all times

$$
\begin{aligned}
\varphi:= & \left(\left|\hat{v}_{a}-28\right| \leq 5\right) \wedge\left(\left|\hat{v}_{b}-28\right| \leq 5\right) \wedge\left(\left|\hat{u}_{a}\right| \leq 8\right) \\
& \wedge\left(\left|\hat{u}_{b}\right| \leq 8\right) \wedge\left(\left|\hat{x}_{a}-\hat{x}_{b}-20\right| \leq 20\right)
\end{aligned}
$$

and $\psi$ specifies a desired gap between the leader vehicles

$$
\psi:=\left(\left|\hat{x}_{a}-\hat{x}_{b}-35\right| \leq 5\right),
$$

which must be attained within $T$ time-steps after the binary signal $S$ becomes equal to 1 . In practice, $S$ could represent another vehicle indicating that it would like to merge in 

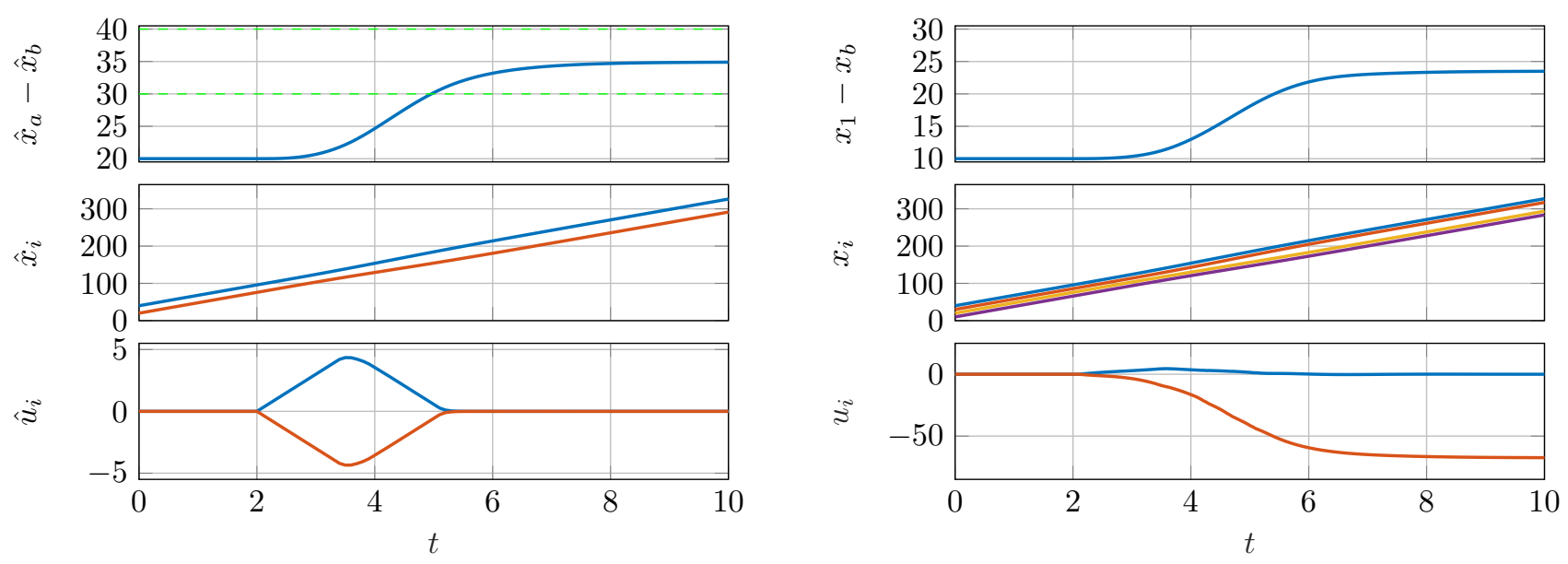

Fig. 2: Simulation results for the platoon separation example. In the abstraction (left), the leader vehicles increase their distance to reach the desired set, indicated by the green dashed lines. The gap maneuver is successfully realized on the full platoon (right), resulting in an increased distance between platoon leader $b$ and follower vehicle 1.

between follower vehicle $n$ and leader vehicle $b$ in the platoon. Using the MPC-based approach in [12], we designed a controller for a time-discretized model of the abstraction which achieves (25).

The constraints in 26 are easily converted to semialgebraic constraint sets $\mathcal{X}$ and $\hat{\mathcal{U}}$. Decision variables $V$ and $\kappa$ are characterized by degree- 2 polynomials with unknown parameters. In this example, $e(0)$ is assumed to be chosen within $\Omega_{\gamma}^{V}$, therefore we can relax 10 to be

$$
\dot{V}(e, \hat{x}, \hat{u})<0, \forall e, \hat{x}, \hat{u}, \text { s.t. } V(e)=\gamma, \hat{x} \in \hat{\mathcal{X}}, \hat{u} \in \hat{\mathcal{U}}
$$

In this case, $V$ becomes a barrier function, and $\Omega_{\gamma}^{V}$ is a forward invariant set for $e(t)$. Consequently, we have a weaker constraint for $s_{3}$ than (14): $s_{3}(e, \hat{x}, \hat{u}) \in \mathbb{R}[(e, \hat{x}, \hat{u})]$. The SOS programming was formulated and translated into SDP using the sum-of-square module in SOSOPT [14] on Matlab, and solved by Mosek [15]. The computation was performed on a workstation with a $2.7[\mathrm{GHz}]$ Intel Core i5 64 bit processor and $8[\mathrm{~GB}]$ of RAM, and this 8 -state example takes around 15 minutes to solve. The simulation results are shown and explained in Figure 2.

\section{Double Pendulum Example}

In this example we consider the fully-actuated double pendulum dynamics, where torques are applied at the both joints. The polynomial dynamics for the double pendulum shown below are obtained by a least-squares approximation of the full equation for $\left(x_{1}, x_{3}\right) \in[-1,1] \times[-1,1]$ :

$$
\left[\begin{array}{c}
\dot{x}_{1} \\
\dot{x}_{2} \\
\dot{x}_{3} \\
\dot{x}_{4}
\end{array}\right]=\left[\begin{array}{c}
x_{2} \\
f_{2}\left(x_{1}, x_{2}, x_{3}, x_{4}\right) \\
x_{4} \\
f_{4}\left(x_{1}, x_{2}, x_{3}, x_{4}\right)
\end{array}\right]+\left[\begin{array}{cc}
0 & 0 \\
8 & -31.2 \\
0 & 0 \\
-31.2 & 391.2
\end{array}\right]\left[\begin{array}{l}
u_{1} \\
u_{2}
\end{array}\right],
$$

$$
\begin{aligned}
& f_{2}=- 3.447 x_{1}^{3}+2.350 x_{1}^{2} x_{3}+1.303 x_{1} x_{3}^{2}+3.939 x_{3}^{3} \\
&+ 21.520 x_{1}-5.000 x_{3}, \\
& f_{4}=4.023 x_{1}^{3}-36.551 x_{1}^{2} x_{3}-4.131 x_{2}^{2} x_{3}-27.060 x_{3}^{3} \\
& \quad-25.115 x_{1}+77.700 x_{3},
\end{aligned}
$$

where $x_{1}$ and $x_{3}$ represent $\theta_{1}$ and $\theta_{2}$ (shown in Fig. 33, which are angular positions of the first and second links (relative to the first link); $x_{2}$ and $x_{4}$ represent $\omega_{1}$ and $\omega_{2}$, which are angular velocities of two links; $u_{1}$ and $u_{2}$ are torques applied at the joint 1 and joint 2 (shown in Fig. 3), respectively.

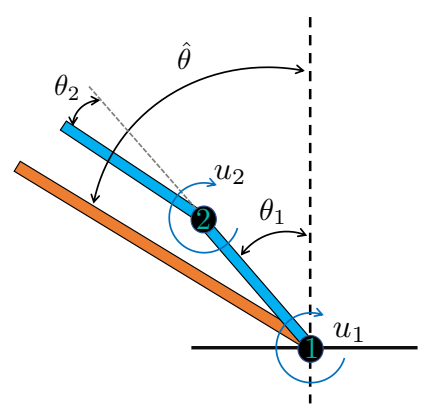

Fig. 3: Double pendulum and its abstraction as a single pendulum. The angular positions are labelled.

The control objective is to bring $\left[\theta_{1} ; \omega_{1}\right]$ from $[-0.5 ; 0.5]$ to $[0.3 ; 0]$ and to maintain it there, while minimizing the relative angular position $\theta_{2}$ and relative angular velocity $\omega_{2}$ between the two links. We choose the single inverted pendulum model as the abstraction to the original dynamics, design controllers for the abstraction and then synthesize the low level controller $\kappa$ to minimize the error bound between the concrete and abstract models. The polynomial dynamics of the abstraction are computed by a least-squares approximation of the full equation for $\hat{x}_{1} \in[-1,1]$ :

$$
\left[\begin{array}{l}
\dot{\hat{x}}_{1} \\
\hat{\hat{x}}_{2}
\end{array}\right]=\left[\begin{array}{c}
\hat{x}_{2} \\
-5.131 \hat{x}_{1}^{3}+32.1 \hat{x}_{1}
\end{array}\right]+\left[\begin{array}{c}
0 \\
9.1
\end{array}\right] \hat{u}
$$


where $\hat{x}_{1}$ represents $\hat{\theta}$ (shown in Fig. 3 , the angular position of the single pendulum, $\hat{x}_{2}$ represents $\hat{\omega}$, the angular velocity, and $\hat{u}$ represents the torque applied at the joint 1 . Based on the control objective, the manifold is chosen as:

$$
x=P \hat{x}, \text { where } P=\left[\boldsymbol{I}^{2} ; \mathbf{0}^{2 \times 2}\right] .
$$

To synthesize the low level control law $\kappa$ and compute the error bound, we enforce the following states and inputs constraints when design the MPC controller for the abstract system

$$
\begin{aligned}
& \hat{x}(t) \in \hat{\mathcal{X}}=\left\{\hat{x} \in \mathbb{R}^{\hat{n}}:\left|\hat{x}_{1}\right| \leq 0.6,\left|\hat{x}_{2}\right| \leq 1.3\right\}, \\
& \hat{u}(t) \in \hat{\mathcal{U}}=\left\{\hat{u} \in \mathbb{R}^{\hat{m}}:|\hat{u}| \leq 5\right\} .
\end{aligned}
$$

$V$ and $\kappa$ are parameterized by degree- 2 and degree- 4 polynomials, respectively. The whole computation including the initialization takes less than 5 minutes to perform on a workstation with a $2.7[\mathrm{GHz}]$ Intel Core i5 64 bit processor and 8 [GB] of RAM. The simulation results are shown in Figure 4. We can see that the error between the phase portrait of $\theta_{1}$ and $\omega_{1}$ and that of $\hat{\theta}$ and $\hat{\omega}$ is small, the phase portrait of $\theta_{2}$ and $\omega_{2}$ stays closely to the origin, and the simulation trajectory of $u_{1}$ is close to that of $\hat{u}$.
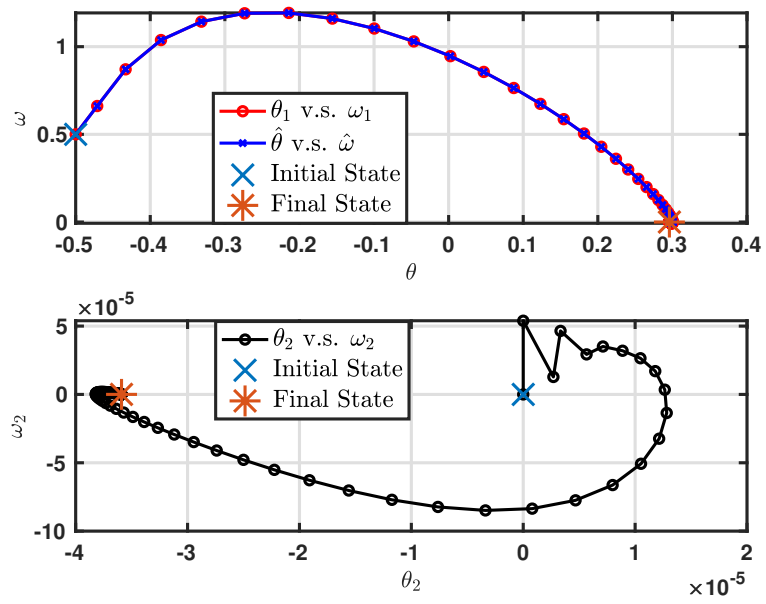

(a) Phase portraits for the double pendulum example

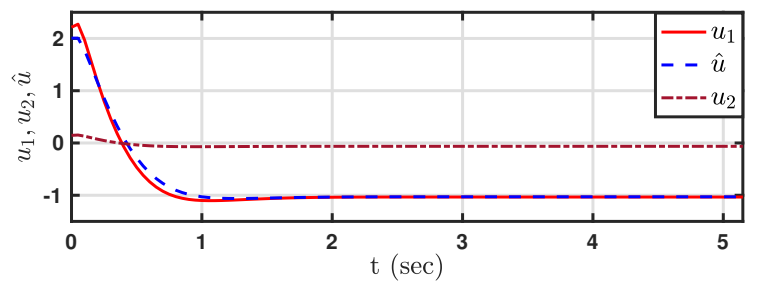

(b) Control inputs vs. time

Fig. 4: Simulation results for the double pendulum example

\section{CONCLUSION}

In this paper we proposed an abstraction-based control design approach for nonlinear control-affine systems. In particular, we utilized SOS optimization to compute a lowlevel controller and a bound on error states between the original system and its abstraction. This optimization procedure assumes that the dynamics are approximated with a polynomial model and the abstract state and input are restricted to semi-algebraic constraint sets. We also proposed an iterative procedure to refine these constraint sets to the necessary level of restrictiveness so that an acceptable error bound is found. Finally, we applied our methods to two examples which demonstrate its broad applicability.

\section{REFERENCES}

[1] C. Belta, B. Yordanov, and E. A. Gol, Formal Methods for DiscreteTime Dynamical Systems. Springer, 2017, vol. 89.

[2] A. Girard and G. J. Pappas, "Hierarchical control system design using approximate simulation," Automatica, vol. 45, no. 2, pp. 566-571, 2009.

[3] M. Rungger and M. Zamani, "Compositional Construction of Approximate Abstractions of Interconnected Control Systems," IEEE Transactions on Control of Network Systems, vol. 5, no. 1, pp. 116127, 2018.

[4] M. Zamani and M. Arcak, "Compositional Abstraction for Networks of Control Systems: A Dissipativity Approach," IEEE Transactions on Control of Network Systems, vol. 5, no. 3, pp. 1003-1015, 2018.

[5] S. W. Smith, M. Arcak, and M. Zamani, "Hierarchical Control via an Approximate Aggregate Manifold," in 2018 Annual American Control Conference (ACC). IEEE, 2018, pp. 2378-2383.

[6] S. W. Smith, M. Arcak, and M. Zamani, "Approximate abstractions of control systems with an application to aggregation," arXiv e-prints, p. arXiv:1809.03621, Sep 2018.

[7] S. L. Herbert, M. Chen, S. Han, S. Bansal, J. F. Fisac, and C. J. Tomlin, "FaSTrack: a Modular Framework for Fast and Guaranteed Safe Motion Planning," in 2017 IEEE 56th Annual Conference on Decision and Control (CDC). IEEE, 2017, pp. 1517-1522.

[8] S. Singh, M. Chen, S. L. Herbert, C. J. Tomlin, and M. Pavone, "Robust Tracking with Model Mismatch for Fast and Safe Planning: an SOS Optimization Approach," arXiv preprint arXiv:1808.00649, 2018.

[9] S. Kousik, S. Vaskov, M. Johnson-Roberson, and R. Vasudevan, "Safe Trajectory Synthesis for Autonomous Driving in Unforeseen Environments," in ASME 2017 Dynamic Systems and Control Conference. American Society of Mechanical Engineers, 2017, pp. V001T44A005V001T44A005.

[10] A. Majumdar and R. Tedrake, "Funnel libraries for real-time robust feedback motion planning," The international Journal of Robotics Research, vol. 36, pp. 947-982, 2017.

[11] O. Maler and D. Nickovic, "Monitoring Temporal Properties of Continuous Signals," in Formal Techniques, Modelling and Analysis of Timed and Fault-Tolerant Systems. Springer, 2004, pp. 152-166.

[12] V. Raman, A. Donzé, M. Maasoumy, R. M. Murray, A. SangiovanniVincentelli, and S. A. Seshia, "Model Predictive Control for Signal Temporal Logic Specification," arXiv e-prints, p. arXiv:1703.09563, Mar 2017.

[13] P. Parrilo, "Structured Semidefinite Programs and Semialgebraic Geometry Methods in Robustness and Optimization," PhD thesis, California Institute of Technology, 2000.

[14] P. Seiler, "SOSOPT: A Toolbox for Polynomial Optimization," ArXiv e-prints, aug 2013, arXiv:1308.1889.

[15] M. ApS, "The MOSEK Optimization Toolbox for MATLAB Manual Version 8.1." 2017, http://docs.mosek.com/8.1/toolbox/index.html 